\title{
Discapacidad, infancias y desigualdades en el Gran La Plata
}

Disability, childhood and inequalities in Gran La Plata

Paula Mara Danel

Licenciada en Trabajo Social

(Universidad Nacional de La Plata)

Especialista en Epistemologías del Sur (CLACSO)

Magíster en Trabajo Social

(Universidad Nacional de La Plata)

Doctora en Trabajo Social

(Universidad Nacional de La Plata)

Investigadora Adjunta CONICET

(Universidad Nacional de La Plata)

Investigadora en Instituto de Estudios

de Trabajo Social y Sociedad

(Universidad Nacional de La Plata)

Correo: danelpaula@hotmail.com 


\title{
Resumen
}

El artículo desarrolla reflexiones generadas en el marco de una investigación sobre discapacidad, infancias y pobreza en el territorio del Gran La Plata, provincia de Buenos Aires, Argentina. En el mismo, se desarrollan intersecciones analíticas sobre trayectorias vitales de niños/as en situación de discapacidad en contexto de pobreza, y se tematiza sobre los procesos de inclusión social de las infancias e intervención social, a la luz de las barreras que producen a la discapacidad. La misma, es pensada y abordada desde el modelo social, incluyendo anudamientos singulares que se efectúan en nuestra región. La investigación se desarrolló con metodología cualitativa y diseño descriptivo. Se efectuó revisión bibliográfica hermenéutica de estudios anteriores, análisis de datos estadísticos, análisis de documentos, observación no participante y entrevistas en profundidad. La investigación mencionada permitió identificar cómo la discapacidad medicalizada y mercantilizada se hace presente en las construcciones de vida de los/as niños/as y en las producciones de los futuros posibles. Además, se pone en evidencia la crianza y cuidados feminizados y las marcas de la desigualdad en los mismos.

\section{Palabras clave}

Discapacidad, Infancias, Desigualdades, Normalización.

\begin{abstract}
The article develops reflections generated in the framework of an investigation on disability, childhood and poverty in the territory of Gran La Plata, province of Buenos Aires, Argentina. In it, analytical intersections are developed on the life trajectories of children with disabilities in the context of poverty, and it is thematized about the processes of social inclusion of children, and social intervention, in light of the barriers that produce disability. It is thought and approached from the social model, including unique issues that take place in our region. The research was developed with qualitative methodology, descriptive and explanatory design $\mathrm{n}$ addition, a hermeneutic bibliographic review of previous studies, statistical data analysis, document analysis, non-participant observation and in-depth interviews were carried out. The aforementioned research allowed us to identify how medicalized and commercialized disability is present in children's life constructions and in the production of possible futures. In addition, feminized upbringing and care and the marks of inequality in them are evidenced.
\end{abstract}

Keywords

Disability, Childhood, Inequalities, Normalization. 


\section{Introducción}

El artículo propone cruces analíticos entre infancia y discapacidad en la pesquisa del proceso de producción de desigualdades de orden económico, generacional, de género y funcional. Se observan procesos de inclusión social de niños, niñas y jóvenes en situación de discapacidad, con sus situados anudamientos y trayectorias singulares.

Se pondrán en escena, algunas experiencias vitales desde el entendimiento que las desigualdades toman rostros con marcas generacionales, entramados con procesos de normalización de las infancias (Barral, Paterson, Stiker, Chauvière, 2000; Pinel e Itard, 1978; Foucault, 2011; Miguez, 2009). Siguiendo con la idea de desigualdades decidimos avanzar en el análisis de barreras, las que se presentan en vinculación a las trayectorias desde las claves analíticas de Castilla García (2019), quien manifiesta que la desigualdad tiene condicionantes en variables estructurales, relacionales, culturales y subjetivas.

Los estudios sobre discapacidad, desde las ciencias sociales, proponen análisis de las relaciones que se producen entre los déficits y los sujetos. Se reconocen diferencias en las matrices analíticas, por un lado, aquellas que destacan la existencia de un funcionamiento corporal (físico, psíquico o sensorial) distinto a los patrones de normalidad que cada formación social produce. Y pone especial atención a las formas de los dispositivos y las relaciones sociales, para identificar si operan como facilitadores de procesos de inclusión o si se constituyen en barreras a la participación social, a la integración y al despliegue de potencialidades. Por otro lado, identificamos matrices que se ocupan de develar cómo los déficits son producto del orden social, que aquello presentado como normal/biológico no es otra cosa que el modo en que una formación social produce performáticas ideas sobre lo que se conoce como natural. Este grupo de intelectuales planteará que esa producción social de déficit genera relaciones de opresión (Oliver, 2002).

La discapacidad es entendida como producto en sociedades capitalistas, con primacía de normalidad (Foucault, 2011) asociada a procesos de producción, reproducción y apropiación desigual de los bienes ponderados como necesarios. Las diversas situaciones de discapacidad adquieren otro 
significado cuando ocurren en contextos de pobreza, tal como afirman varias investigaciones (Vallejos, 2005; CEPAL, 2010; Grech, 2016).

La Convención de los Derechos de las Personas con Discapacidad (de ahora en más CDPD) (Naciones Unidas, 2006) señala que

La discapacidad es un concepto que evoluciona y que resulta de la interacción entre las personas con deficiencias y las barreras debidas a la actitud y al entorno que evitan su participación plena y efectiva en la sociedad, en igualdad de condiciones con las demás (2006:1).

A partir de la Convención, emergen discusiones que ponen en tensión las formas de nombrar a las personas -consideradas dentro de este grupo- $y$, al mismo tiempo, discute si el consenso arribado en el instrumento jurídico resulta suficiente para producir procesos de transformación.

En esa línea, se inscribe la presente investigación habida cuenta que posibilitó adentrarse en la trama cotidiana de producción de barreras, de limitaciones al cumplimiento de derechos para este grupo poblacional, de generación y de clase.

Uno de los tópicos que abordamos, estuvo relacionado a los efectos perfomativos del nombrar y del establecimiento de diagnósticos.

(...) el diagnóstico es, como la medicalización, un fenómeno social que puede analizarse más allá de lo estrictamente médico o psiquiátrico (si es que ello existe). El diagnóstico admite ser abordado tanto en sus raíces histórico sociales como en sus efectos performativos, ya que él puede considerarse una expresión cultural de aquello que la sociedad acepta como normal y lo que considera que debe ser tratado médicamente. (Barcala, Bianchi y Poverene, 2017:105).

El diagnóstico en discapacidad, especialmente en la infancia, coloca en escenarios difusos, opacos en los que la tensión entre los procesos de crecimiento y devenir propio de la infancia colisiona con las rigideces de los diagnósticos. Se afirma que la discapacidad mirada, intervenida y corporizada por niños y niñas, se instituye en espacios de borde, liminares entre las lógicas jurídicas progresistas, experiencias situadas de restricciones y, tal vez, 
experiencias vívidas de infancias. Interesa recuperar estas tensiones en diálogo con los estudios sociales sobre infancias, y advertir que la ideología de la normalidad (Oliver, 2002) es la que pretende demarcar lo que se construye como socialmente aceptable:

(...) el telos de la infancia es su direccionalidad determinada para ser adulto. Ese recorrido está "normalizado" generalmente en una curva de crecimiento cuyo recorrido es universal. La socialización de la subjetividad consiste en su adaptación a un desarrollo "normalizado". Se pasa de un estado pre social a un estado social, y los desvíos son conceptualizados en términos de varianza y márgenes de error (Bustelo, 2012:289).

La normalización de las crianzas, socializaciones e interrelaciones se conectan con otras experiencias educativas, familiares, institucionales en las que la emancipación se busca de forma incesante (Walsh, 2013); "La administración de la infancia es un sitio crucial donde se juega la tensión entre reproducción y transformación social. Conjuga las dimensiones pedagógicas y represivas del Estado" (Llobet, 2019:29).

Con esto señalamos que la infancia es devenir, en la misma se desarrollan aprendizajes, y está moldeada por estrategias estatales. En ese punto, los aportes de Vianna (2010) invitan a pensar cómo los Derechos del Niño, Niña y Adolescentes producen retóricas que operan como amplios repertorios discursivos. Los mismos resultan ordenadores de las prácticas, acciones, miradas sobre las infancias. La conformación de frentes discursivos (Fonseca y Cardarello, 1999) se constituye en herramienta para comprender los modos en que la categoría de derechos e infancias generan impregnación y visibilidad. El frente discursivo, el repertorio discursivo, en tanto productos socio- culturales están puestos en disputa, sujetos a intercambios e impugnados desde las prácticas. Los Derechos de niños y niñas imponen terminologías técnicas que amplían sus efectos en otros espacios (Barna, 2012). Los sentidos cristalizados en ellos, están contextualmente construidos y reñidos por diversos actores, lo que se complejiza -en situaciones como las investigadas- con las disputas en torno a derechos generacionales (de infancias) y de condición (discapacidad). 
También, la Convención de Derechos de las Personas con Discapacidad (Naciones Unidas, 2006) sustenta la imagen de que los niños/as con discapacidad deben gozar de los derechos humanos y las libertades fundamentales en igualdad de condiciones que los demás, y coloca a los estados como los obligados para el cumplimiento de esos derechos.

Barna (2013) manifiesta que las retóricas de los derechos del niño, y agregamos de las niñas con discapacidad, se cristalizaron en legislaciones globales y propone reflexiones para desentrañar las tensiones que están presentes en esos criterios universales. Grinberg (2013) analiza el contexto de recepción de la Convención Interamericana de Derechos de Niños/as y Adolescentes (CIDN) y los fructíferos vínculos con los movimientos de Derechos Humanos. En el caso de la convención de discapacidad ${ }^{1}$ también resulta fundamental la observancia del contexto y las relaciones con los movimientos asociativos.

En los 30 años de vigencia de la CIDN y los 12 años de la Convención de Discapacidad, es necesario develar los efectos en las trayectorias singulares. Barna (2013) destaca que dichos efectos resultan de intensidad variable en cada país, aunque se ha logrado imponer en el caso de la CIDN "la idea del niño y sus derechos" (Barna, 2013:24). El mismo autor advierte sobre el riesgo de reificar y dicotomizar los derechos, ya que no se trata de pensar en ideales -constantemente-, sino de tramar esos derechos en tanto herramientas. Ahora bien, la idea de derechos sacralizados desde la observación de trayectorias sociales de niños/as en situación de discapacidad nos permite pensar las inaccesibilidades como forma de opresión, pensar que las mismas son producto de los modos patriarcalmente organizados de las crianzas/ cuidados y de las particularidades que asumen las intervenciones sociales del Estado.

1 Para ampliar sobre este tema, sugiero: http://portal.uc3m.es/portal/page/portal/instituto derechos humanos/conferencia cdpd 


\section{Metodología: acerca del territorio en el que se realizó la investigación}

La investigación se desarrolló con metodología cualitativa y diseño descriptivo. Se mantuvo durante todo el proceso de investigación una posición exploratoria, de interrogación y de apertura. Se efectuó revisión bibliográfica hermenéutica de estudios anteriores, análisis de datos estadísticos, análisis de documentos, observación no participante y entrevistas en profundidad. La misma se ha desarrollado en el área poblacional denominada Gran La Plata, en la Provincia de Buenos Aires, Argentina. El mencionado aglomerado urbano está conformado por los distritos de La Plata, Berisso y Ensenada. La región es desigual, con mayor concentración de pobreza en las periferias de las tres ciudades que la conforman (Alzugaray, Santa María y Peiró, 2019). Por su parte, La Plata es la que acumula la mayor cantidad de organizaciones de los estados nacional y provincial.

Los principales rasgos de la estructura socioeconómica del Gran La Plata están dados por el significativo peso del sector terciario, en particular el sector público, que se concentra fundamentalmente en la ciudad de La Plata, dado su carácter de capital provincial, sede de la Universidad Nacional y de numerosas entidades empresariales y financieras; y por la importancia de las grandes industrias de las ramas metalmecánica y petrolera emplazadas en el área portuaria: destilería y petroquímica Repsol-YPF, astilleros Río Santiago y siderurgia SIDERAR. Las micro, pequeñas y medianas industrias se concentran en las ramas alimenticias, metalmecánicas y textiles destinadas al mercado local y se localizan principalmente en la trama urbana del aglomerado. El empleo industrial, por su parte, se destaca en Berisso y Ensenada pues en ambos partidos ocupa el segundo lugar; mientras que en La Plata ocupa el tercero, aunque agrupando el $74 \%$ del total de los ocupados en la industria (Adriani, Pintos y Suárez, 2011:21).

Las características económicas asentadas en la producción, brindan pistas para analizar las formas que asumen los tránsitos en la ciudad. Es decir, el modo en que gravita la presencia de circuitos, itinerarios y accesibilidades. Para el tema de estudio, nos interesa identificar cómo la discapacidad se hace presente en el espacio público y de qué forma los niños/as con discapacidad acceden a las prestaciones, instituciones y servicios que necesitan: 
Pensar las desigualdades socio-espaciales supone, entonces, situarse "más acá” de la segregación y de los grupos segregados. Implica reconocer simultáneamente tanto la importancia de las desiguales posiciones socio-espaciales de los agentes en la ciudad (y su eventual territorialización) así como las movilidades que se producen en (y, eventualmente, pueden alterar) dichos territorios. La cambiante y heterogénea articulación entre (des) territorializaciones e (in)movilidades cotidianas en la ciudad nos coloca ante un escenario que asume la forma no tanto de la oposición entre lo incluido y lo excluido, sino de desiguales velocidades y posibilidades de acceso a la ciudad y sus beneficios (Segura y Chaves, 2019:203).

Por ello, el trabajo asume una mirada del territorio con sus transacciones, articulaciones y los modos en que se hace posible el ingreso a la ciudad, o mejor dicho a los recursos que la misma posibilita. Una de las cuestiones que resuena en los relatos de los/as niños/as y sus madres ${ }^{2}$ es que llegar a La Plata resultaba algo no tan posible, ni cotidiano. Al mismo tiempo develaba que las dinámicas de inclusión-exclusión resultan más complejas que sólo pensar en adentro y afuera, habida cuenta que las accesibilidades de las infancias en situación de discapacidad a determinados servicios y recursos, supone cierta domesticación normalizada, patologizada y etiquetada (Miguez, 2009; Bianchi, 2011).

Esas desigualdades moldean las formas que asumen las realidades de niños y niñas, y se entretejen de manera tensa con las ideas sobre derechos y con las temporalidades en juego. La investigación ${ }^{3}$ retoma las trayectorias de personas en situación de discapacidad de todas las edades, por lo que para la presente producción pusimos el foco en las situaciones de niños, niñas y jóvenes con los que se establecieron vínculos en el trabajo de campo.

2 Esta referencia a las madres, si bien será retomado a lo largo del trabajo, amerita reflexiones específicas en torno a la organización social y política del cuidado. Recomendamos el texto de Corina Rodríguez Enrique y de Gabriela Marzonetto (2015). Organización social del cuidado y desigualdad: el déficit de políticas públicas de cuidado en Argentina. Revista Perspectivas de Políticas Públicas, (8).

3 En referencia a la investigación "Las dinámicas de inclusión / exclusión social de las personas en situación de discapacidad y los modos sociales de abordaje en las organizaciones del campo de la discapacidad en el Gran La Plata" que desarrollo en el marco del CONICET. 
Retomando los aportes de Rita Segato (2016), señalamos que la búsqueda en el trabajo de campo estuvo asociada a producir diálogos de saberes. ¿Quiénes más pueden conocer las formas particulares que asumen las experiencias de discapacidad si no son quienes encarnan una identidad atribuida por el estado y los equipos interdisciplinarios? ¿Acaso las barreras del entorno no se hacen carne en cada trámite que no se logra culminar, porque se burocratiza?

La investigación que enmarca esta propuesta tiene como objetivo conocer las lógicas, prácticas y sentidos vigentes en los abordajes hacia las personas con discapacidad, así como producir procesos de reflexividad con los agentes involucrados en las mismas. Por ello, es que compartimos referencias desde un corpus de investigación configurado por 10 (diez) entrevistas desarrolladas durante el último trimestre 2019 y el primero del 2020, sumado al acceso, durante el segundo semestre 2019, a documentación de organismos públicos provinciales y municipales sobre 15 situaciones de niños/as y jóvenes. Los criterios de selección de entrevistados han sido diferenciales de acuerdo a la estrategia. Por un lado, las unidades domésticas con las que se realizaron observaciones $\mathrm{y} / \mathrm{o}$ entrevistas presentaban entre sus miembros a niños, niñas o jóvenes en situación de discapacidad, y sostenían relaciones con el estado para el acceso a prestaciones específicas. Para el acceso a los documentos, y registros de los casos, se establecieron contactos con organismos ${ }^{4}$ públicos, siendo el análisis efectuado de manera interpretativa, y contextualizada. En cuanto a las entrevistas a profesionales de las áreas de discapacidad municipal y otros organismos, fueron realizadas con guiones unificados, priorizando la realización de entrevistas a trabajadoras sociales.

En relación a los datos estadísticos, compartimos (Cuadro 1 y 2) aquellos que surgen del Censo Nacional 2010 y señalamos que el universo de niños/as con discapacidad en el aglomerado Gran La Plata es menor a los otros grupos de edad y rondaba, al momento del censo, un número superior a 9000 niños, niñas y adolescentes en situación de discapacidad.

4 Se accedió a documentación de la Secretaría de Políticas de Género, Niñez y Adolescencia de la Defensoría del Pueblo de la Provincia de Buenos Aires, en el año 2019 y a registros de los Municipios integrantes del Gran La Plata. 
Cuadro 1. Población en viviendas particulares con dificultad o limitación permanente por cantidad de dificultades o limitaciones permanentes, según sexo y grupo de edad por municipio.

\begin{tabular}{|c|c|c|c|c|c|c|c|c|c|}
\hline \multicolumn{2}{|c|}{$\begin{array}{l}\text { Pcia. de Buenos Aires } \\
(2010)\end{array}$} & & & & & & & & \\
\hline \multirow[t]{2}{*}{ Municipio } & \multirow{2}{*}{$\begin{array}{l}\text { Población } \\
\text { en vivien- } \\
\text { das parti- } \\
\text { culares con } \\
\text { limitación } \\
\text { permanen- } \\
\text { te }\end{array}$} & \multicolumn{4}{|l|}{ Con una } & \multicolumn{4}{|c|}{ Dos o más } \\
\hline & & Total & $0-14$ & $15-64$ & $\begin{array}{l}65 \mathrm{y} \\
\text { más }\end{array}$ & Total & $0-14$ & $15-64$ & $\begin{array}{l}65 \mathrm{y} \\
\text { más }\end{array}$ \\
\hline $\begin{array}{l}\text { Total Provin- } \\
\text { cia }\end{array}$ & 1.853 .253 & 1.274 .155 & 113.699 & 813.279 & 347.177 & 579.098 & 30.783 & 263.212 & 285.103 \\
\hline BERISSO & 10.216 & 7.419 & 571 & 4.832 & 2.016 & 2.797 & 148 & 1.141 & 1.508 \\
\hline ENSENADA & 6.523 & 4.433 & 452 & 2.713 & 1.268 & 2.090 & 107 & 1.004 & 979 \\
\hline LA PLATA & 65.235 & 45.489 & 3.395 & 28.465 & 13.629 & 19.746 & 720 & 8.127 & 10.899 \\
\hline
\end{tabular}

Fuente: Censo Nacional 2010 - INDEC.

Cuadro 2: Niños, niñas y adolescentes con alguna dificultad o limitación permanente por tipo de dificultad o limitación permanente. Provincia de Buenos Aires. Año 2010

\begin{tabular}{|l|l|l|l|}
\hline Municipio & & & \\
\hline & \multirow{2}{*}{ Total } & \multicolumn{2}{|l|}{ Con alguna dificultad } \\
\cline { 3 - 5 } & & Absoluto & $\%$ \\
\hline Total Provincia & & & \\
\hline BERISSO & & $\mathbf{2 5 1 . 5 7 2}$ & $\mathbf{5 , 4}$ \\
\hline ENSENADA & $\mathbf{4 . 6 4 3 . 5 7 3}$ & $\mathbf{1 . 2 5 7}$ & $\mathbf{4 , 8}$ \\
\hline LA PLATA & $\mathbf{2 6 . 0 5 2}$ & $\mathbf{8 4 8}$ & \\
\hline & $\mathbf{1 7 . 5 9 0}$ & & \\
\hline
\end{tabular}

De los datos de la Dirección General de Escuelas de la Provincia de Buenos Aires surge que la matrícula de la modalidad educación especial representa el 1,8\% de la matrícula total de alumnos de la Provincia de Buenos Aires. Y también destacan que "De la matrícula integrados (sólo apoyo) del total de la provincia, el sector de gestión estatal tiene un $83 \%$ de estudiantes integrados en todos los niveles. El sector de gestión privada, el 16,25\%, (...). La 
discapacidad intelectual tiene la mayor representación porcentual en el total provincial y en todos los sectores de gestión” (DGCyE, 2019:10)

De acuerdo al análisis de matrícula y establecimientos educativos surge la siguiente información:

Cuadro 3: Elaboración propia a partir de datos estadísticos de anuario (DGCyE, 2018).

\begin{tabular}{|l|l|l|l|}
\hline & Berisso & Ensenada & La Plata \\
\hline Matrícula total de distrito (en todas las modalidades) & 22606 & 13034 & 170401 \\
\hline Establecimientos educativos todas las modalidades & 77 & 49 & 579 \\
\hline Matrícula modalidad educación especial & 385 & 129 & 3451 \\
\hline Establecimientos modalidad especial & 2 & 1 & 28 \\
\hline Matrícula que recibe algún servicio alimentario escolar & 324 & 110 & 2819 \\
\hline
\end{tabular}

Los datos compartidos nos permiten caracterizar un complejo universo de dispositivos educativos en los que las infancias en situación de discapacidad de nuestra región, transitan. Y el número de niños/as escolarizados en modalidad especial en la región ascendía, al 2018, a 3965.

Como se ha señalado, las infancias en situación de discapacidad en contexto de pobreza, se instalaron como preocupación epistémica y ética, por lo que desplegamos instrumentos complementarios como la reconstrucción de la territorialidad, desde la identificación de prácticas urbanas -trayectos, circuitos, usos y límites socioespaciales- de las personas en situación de discapacidad (Segura, 2009 y 2013). Se buscó dar cuenta de la materialidad que adquieren las distancias sociales, realizando observaciones participantes en los escenarios de atención social, buscando caracterizar las dinámicas de atención e identificar los obstáculos presentes en la misma.

Para dar cuenta de la producción social de las infancias, en su intersección con la discapacidad, avanzamos en incluir el componente narrativo

Las narrativas nos permiten adentrarnos a las formas en que se producen sentidos a partir de lo que las personas dicen, de sus modos de simbolizar y transmitir mediante las expresiones verbales, sus teorías sobre aquello que les acontece, las relaciones con otros sujetos y la posibilidad de tomar decisiones sobre sus propias trayectorias vitales. Las narrativas nos permiten es- 
tablecer relaciones con las biografías personales y el lugar otorgado a aquello que los hace vincular al trabajo social (Danel y Sala, 2019:87).

Esas narrativas fueron producidas por madres, niños/as, y profesionales del Trabajo Social, dialogadas, entrelazadas y argumentadas de manera recíproca.

\section{Resultados: las situaciones de niños/as en el trabajo de campo}

El procesamiento analítico desarrollado, ha permitido configurar, identificar los circuitos que caracterizan las situaciones de niñas/os ${ }^{5}$ en las que se trama discapacidad y pobreza. Se trata de una categorización, desde dinámicas territoriales situadas, de procesos de inclusión-exclusión y de multiplicidades en la producción de las infancias. En tal sentido, proponemos algunas líneas que, si bien no son excluyentes, delinean algunos de los circuitos que más marcas subjetivas y materiales producen en las trayectorias.

Los circuitos que transitan los/as niños en situación de discapacidad están vinculados a los esfuerzos socio-familiares para el acceso a la educación, a prestaciones de apoyo o rehabilitación y a sostener la crianza en el seno de los hogares. Destacamos que, en las situaciones analizadas la discapacidad no resultaba el único vector en la producción de barreras. En la mayoría de los casos se producía un entramado entre pobreza urbana, feminización de las crianzas, acciones estatales de culpabilización (emergencia de la idea de negligencia) y mercantilización de las prestaciones.

En esa línea, proponemos un agrupamiento de las situaciones de niños/ as que ven tensionado el acceso a la educación en referencia a las situaciones en las que la presencia en el espacio escolar es realizada en un espacio de opacidad entre aprendizajes efectivos, presencias ignoradas y desigualdades en el acceso a las prestaciones apoyo. Siguiendo los aportes de Kaplan (2006), Yuni y Meléndez (2017) señalamos que la gramática escolar genera procesos de inclusión excluyente y tensa con las lógicas programáticas de

5 Los nombres han sido cambiados a fin de evitar la identificación de los/as niños/as, las entrevistas se realizaron con los consentimientos informados y la documentación que se analizó fue con autorización de las autoridades provinciales y municipales. 
políticas socio-educativas. En este punto afirmamos que del trabajo de campo surgen experiencias de inclusión-excluyente en las que el foco de exclusión se sustenta en los diagnósticos como modo explicativo absoluto de las experiencias educativas.

También se identificaron estudiantes de Escuela Primaria que contaban con un asistente externo o acompañante externo (Resolución 782/13 de Prov. Buenos Aires), lo que viabilizaba (de modo complementario) estrategias de aprendizaje e interacción social. Los apoyos eran cubiertos por sus obras sociales (sistema de seguridad social), especialmente IOMA (obra social provincial) y PAMI (INSSJyP). Las diferencias en el acceso a los apoyos, está asociado a variables estructurales, relacionales, culturales, y subjetivas. Desde el convencimiento de que la inclusión educativa de niños/as está ligada a la disposición en tiempo y forma de los apoyos, podemos afirmar que las experiencias educativas resultan desiguales por no garantizarse de manera igualitaria los apoyos mencionados.

Podemos afirmar que en nuestra región está tensado el derecho a la educación de niños/as en situación de discapacidad en contexto de pobreza que carecen de cobertura de seguridad social y expresada en las gramáticas escolares excluyentes. Coincidiendo con la investigación de Borzi, Sánchez Vázquez y Talou (2019) en que la inclusión requiere de cambios en las perspectivas, en las políticas y en el desarrollo de prácticas inclusivas. Los datos producidos dialogados con otras investigaciones, también de la región (Schwamberger y Grinberg, 2020; Cobeñas, 2020), han permitido identificar cómo las escuelas habitan las tensiones entre ser escuela colador, excluyente y, al mismo tiempo, ser dispositivo de atención de las complejidades, de producción de amorosidad y contención.

Otro de los agrupamientos propuestos está relacionado a los/as niños/ as que encuentran barreras para el acceso a las prestaciones de apoyo o rehabilitación. Nos referimos a situaciones en las que la inaccesibilidad se traduce en limitaciones en la funcionalidad, por lo que claramente se trata de situaciones evitables. Las prestaciones del sistema de salud, destinadas a personas en situación discapacidad, están moldeadas por los preceptos del Sistema Único de Prestaciones Básicas en favor de las personas con 
discapacidad (Ley 24901/97), lo que supone una preeminencia del modelo rehabilitador, y un moldeamiento de las trayectorias conforme el entramado de estos dispositivos. Este conjunto de prestaciones y de respuestas preestablecidas, si bien ha hegemonizado el diseño institucional, no cubre en la región estudiada, las trayectorias de los sujetos carentes de obra social. Identificamos aquí una tensión entre la hegemonía mercantilizada y las desigualdades invisibilizadas.

Las situaciones identificadas denotan, por un lado, la carencia de cobertura de seguridad social, así como carencia de efectores públicos de manera accesible en todo el territorio (Borzi, Talou, Sánchez Vázquez, Hernández Salazar, Gómez y Escobar, 2013) y el incumplimiento de las obras sociales en la prestación efectiva de las prestaciones:

A las prestaciones que necesita, no accede a través de la obra social ya que la distancia entre su domicilio y los lugares de atención es excesiva. Relata que para acceder a la evaluación de psicopedagoga decidió llevarla a un consultorio particular a unas 30 cuadras de su casa (notas de entrevista a mamá de niña de 8 años).

Otra cuestión central está relacionada al acceso a la certificación estatal de la discapacidad, CUD (Certificado único de discapacidad) que se realiza en las sedes municipales y en algunos hospitales provinciales. El tiempo que demora la gestión del CUD entre el establecimiento del diagnóstico médico y la certificación propiamente dicha, en los casos relevados, supera los dos años. De acuerdo a los datos proporcionados por la ANDIS (2018) a nivel nacional del total de certificados emitidos, el 27\% de los mismos se otorgan a menores de 15 años. Siguiendo con los datos construidos en nuestra región destacamos que no se garantiza la trazabilidad en los tratamientos. Como ejemplo, destacamos que en los casos en que pierden un turno son las mismas familias las que deben retomar el contacto. Aquí coincidimos con Villalta (2019) quien refiere que las producciones sobre la infancia pobre están asociadas a las ideas de riesgo y negligencia, al tiempo que de cuidado y tutela. Por lo tanto, las familias asumen de modo precario la sustentabili- 
dad de cuidados y tratamientos, en varias situaciones sin los apoyos estatales adecuados.

Fonseca y Cardarello (1999) plantean que se produjo un deslizamiento semántico desde las ideas de problema socio-económico hacia la de negligencia, las familias pobres resultan culpabilizadas por la situación en la que se encuentran sus hijos/as. Esto lo identificamos con claridad en las valoraciones hacia las madres, principales referentes de los cuidados parentales.

En este punto, proponemos otro de los agrupamientos los/as niños/as en situación de discapacidad que tienen rigidizado el acceso a los cuidados parentales. En el mismo incluimos situaciones de niños/as y jóvenes que transitan su cotidianidad en dispositivos residenciales (hogares) del sistema de promoción y protección integral (Ley 13298) y que cuando son mayores de 18 años egresan (algunas veces) sin los entramados que posibiliten su vida en comunidad con proyectos progresivamente autónomos (Favero Avico, 2019). Las situaciones relevadas van desde acciones judiciales en las que prohíben el contacto con la familia de origen, a la indiferencia absoluta a madres de niños con discapacidad en lo que respecta a la configuración de apoyos. Esto lo relacionamos con lo identificado por Rivas et al (2019) en torno al incremento del pedido de vacantes en hogares, durante la gestión gubernamental 2015-2019, poniendo en evidencia una tendencia a propiciar la separación de los niños de su familia de origen e institucionalizarlos.

Cuando las madres claudican en los cuidados (por muerte, enfermedad u otras circunstancias) resultan otras mujeres las que asumen los mismos. Identificamos situaciones en las que los cuidados pasan de madres a tías o abuelas. Una situación a destacar es la de un joven que asiste, a través de su obra social de lunes a viernes al dispositivo "Centro de Día” de gestión privada. Los sábados participa de un espacio grupal que sostiene el municipio. Los equipos interdisciplinarios que están en contacto con el joven manifiestan que se produce abandono y debilidad de los cuidados parentales. "Los fines de semana queda descubierto (...) sabemos que el Domingo no tiene nada" (Trabajadora social entrevistada).

En otras ocasiones la falta de cuidados parentales se asocia a la situación de discapacidad de las madres. Las mujeres, madres con discapacidad, son 
colocadas a demostrar constantemente su capacidad de desarrollo de cuidados.

Los cuidados parentales, se traman de manera sutil en un espacio patriarcalmente moldeado y al mismo tiempo como espacio privilegiado de producción del lazo social. Los cuidados en la infancia, resultan sustantivos al tiempo que los mismos son mayoritariamente desarrollados con manos de mujeres. Referenciamos la idea de rigidez, en tanto el acceso a los cuidados presenta dificultades, miradas estatales culpabilizantes y labilidad en las respuestas a las necesidades de las unidades domésticas por parte de las organizaciones estatales. Esto se suma a la tendencia mercantilizadora de la producción de dispositivos residenciales en el marco del Sistema único de prestaciones que mencionamos en el apartado anterior.

Finalmente, destacamos que todos/as los/as niños/niñas transitan sus infancias más allá de las etiquetas de la discapacidad. Si bien opera con fortaleza la marca des-subjetivizante de los abordajes de la discapacidad, la misma no logra desaparecer lo vívido de la infancia. Skliar (2009) nos invita a pensar en infancias, reales, con barreras, con experiencias heterogéneas, felices, sufrientes. Los/as niños/as no debieran ser pensados/as sólo cómo personas con discapacidad, o con dificultades de aprendizaje, o pobres, o residentes en contexto de precariedad. Son niños/as con derechos, que se instalan en escenarios discursivos de disputa.

Algunos/as de los/as jóvenes entrevistados/as han construido lazos sociales por fuera de los circuitos exclusivos de la discapacidad, y aún así requieren de apoyos oportunos. Las historias relevadas permiten identificar cómo las experiencias vitales de niñas, niños y jóvenes son desiguales, lo que lleva a la noción de pobreza en el marco de esas desigualdades. Se relacionan los desarrollos conceptuales de Gutiérrez (2004) para pensar esas desigualdades, la pobreza y el modo singular en que se expresa la condición infantil en las situaciones de discapacidad (Acuña, Chávez Asencio, Calfunao, Urrutia y Danel, 2021).

El discurso experto produce a las infancias normales, y al mismo tiempo traza los espacios por los que pueden transitar: una niña es alumna de una "escuela normal", su madre refiere que por su condición no estará mucho 
tiempo allí ya que "no ha logrado aprender a leer y escribir". Los hermanitos pobres, cuya mamá presenta déficit intelectual y requiere apoyos para la crianza, en vez de disponerlos desde el estado, se decide llevar a los niños a un hogar de gestión privada por el que abona un alto costo. El jovencito que fue expulsado de una escuela que no fue capaz de configurar apoyos, la joven que asiste a un centro de día privado por el que la obra social abona 25 veces lo que el joven percibe como pensión. Otra niña ha sido intervenida de manera incesante durante sus primeros años, y una vez que acreditaron cuál era su condición de salud limitaron los acompañamientos, las miradas atentas, las estrategias de intervención. Los tránsitos escolares parecen desapegados.

La desigualdad expresada en trayectorias "de como sî", dan pistas para pensar en los/as niños/as, en sus dificultades de aprendizaje y de sostenimiento de la escolaridad. Cuando las mamás reclaman que la escuela no mira a sus hijas/os, o que el hospital no hace seguimiento y que la obra social no responde, surge un enlace a las provocaciones conceptuales de Dubet (2015) quien señala que la elección de la desigualdad no es solamente ideológica, sino que responde a tensiones en la distancia entre el principio de igualdad y las desigualdades sociales materializadas.

La discapacidad medicalizada y mercantilizada (Danel, 2018) es una marca persistente en los abordajes de las infancias con las que interactuamos. La disputa por los derechos nos lleva a pensar en la producción de vidas con dignidad, acceso a educación inclusiva, atención integral de salud y participación en actividades económicas, políticas y culturales (Sala, Pucci y Chávez Asencio, 2020).

Cuando se piensa la discapacidad, anidada a la normalización, a partir de historias vitales, de narrativas encarnadas, contemplando los discursos oficiales, se visualiza y advierte que las infancias en situación de discapacidad no son homogéneas, ni guiones trágicos de historias por venir. Infancias y juventudes plurales que, intersectadas con la discapacidad, ponen en escena historias de opresiones, de inaccesibilidades y al mismo tiempo de infancias vívidas. 


\section{Conclusiones y discusiones}

El trabajo entrelazó debates sobre pobreza, desigualdades y experiencias vitales de niños y niñas etiquetadas bajo la discapacidad. Se ratifican ideas en torno a que la discapacidad es un concepto (Favero Avico y Danel, 2014) y se suman reflexiones sobre la noción de infancias. Las historias aquí compartidas ayudan a adentrarnos en una compleja red de sentidos, que de forma persistente exhibe una mirada médica hegemónica para el abordaje de la discapacidad, iniciada en el establecimiento del diagnóstico.

Otra cuestión sustantiva es la perseverante presencia de familias feminizadas, que enlazan con la idea de feminización de los cuidados. Las madres asumen el lugar de interlocución con las organizaciones estatales, con los equipos interdisciplinarios, lo que podría ser pensado desde las múltiples relaciones que se desarrollan y encuadran como estrategias de los sectores tipificados como pobres.

El orden patriarcal se hace presente en la evaluación de las familias y las infancias que asisten a las instituciones estatales. Las ideas asociadas a negligencias operan como interpelación de las maternidades, y activan como respuestas procesos de capturación de los cuerpos de niños y niñas en circuitos mercantilizados (algunas veces).

Los agrupamientos propuestos: aquellos que experimentan tensiones en el acceso a la educación; los que se encuentran con barreras para el acceso a prestaciones y aquellos que ven rigidizado el acceso a cuidados parentales permiten identificar los modos singulares en que se produce la dinámica de cumplimiento-incumplimiento de los derechos que asisten a niñas/os y al mismo tiempo inscribir en el espacio territorial las barreras, las limitaciones del contexto y las expectativas que los equipos producen en torno a estos grupos.

Otra cuestión emerge en torno a las ideas de futuro, cuando la mamá espera que sus hijos vuelvan al hogar materno, que sean liberados del encierro, está apostando al futuro. Cuando otra madre se lamenta porque la condición de salud de su hija la coloca en un lugar de exclusión educativa, está pensando en el futuro. 
¿Cómo se construye el futuro en sociedades que discapacitan y producen barreras para los desarrollos personales de niños, niñas y jóvenes? Milton Santos (2000) nos invita a pensar la propia existencia como paradoja, es decir, obedecer para subsistir y resistir para poder pensar en el futuro.

Las existencias producen las condiciones para resistir, a las normalizaciones, las exclusiones y las sospechas sobre las madres pobres, sobre los/as niños/as en situación de discapacidad y sus posibles desarrollos personales y colectivos.

\section{Referencias bibliográficas}

Acuña, Araí; Chávez Asencio, Bárbara; Calfunao, Clarisa; Urrutia, Liliana y Danel, Paula (2021). "Infancias, pobreza y discapacidad: análisis interseccional desde la provincia de Buenos Aires, Argentina”. En P. Danel, B. Pérez Ramírez y V. Yarza de los Ríos (Eds.) ¿Quién es el sujeto de la discapacidad?: exploraciones, configuraciones y potencialidades. CLACSO y Facultad de Trabajo Social UNLP, La Plata, Argentina. . Recuperado de: http://biblioteca. clacso.edu.ar/clacso/gt/20210505054147/Quien-es-el-sujeto-de-la-discapacidad.pdf

Alzugaray, Lucas; Santa María, Juliana y Peiró, María Laura (2019). Cartografía del nivel socioeconómico de los hogares del Gran La Plata: Aplicación de un indice a partir de datos del Censo 2010. Universidad Nacional de La Plata, Ensenada, Argentina. Recuperado de http://www.memoria. fahce.unlp.edu.ar $/$ library? $\mathrm{a}=\mathrm{d} \& \mathrm{c}=$ libros \&d$=\mathrm{Jpm} 1070$

Adriani, Héctor; Papalardo, María; Pintos, Patricia y Suárez, María (2011). Actores, estrategias y territorio: El Gran La Plata: De la crisis de la convertibilidad al crecimiento económico. Universidad Nacional de La Plata, La Plata, Argentina. Recuperado de: http://www.memoria.fahce.unlp. edu.ar/libros/pm.288/pm.288.pdf

Agencia Nacional de Discapacidad (ANDIS) (2018). Registro Nacional de Personas con Discapacidad de la República Argentina. Anuario 2018. Recuperado de: https://www.argentina.gob.ar/ sites/default/files/anuario estadistico nacional. ano 2018 0.pdf

Barcala, Alejandra; Bianchi, Eugenia y Poverene, Laura (2017). Medicalización de la infancia: sus efectos en la salud mental. Derecho de Familia. Revista Interdisciplinaria de Doctrina y Jurisprudencia, (82), 99-113.

Barna, Agustín (2012). Convención Internacional de los Derechos del Niño Hacia un abordaje desacralizador. KAIROS. Revista de Temas Sociales. http://www.revistakairos.org

Barna, Agustín (2013). Los derechos del niño. Un campo en disputa. Boletín de Antropología y Educación, (5), 21-25. http://www.derechoshumanos.unlp.edu.ar/assets/files/documentos/ los-derechos-del-nino-un-campo-en-disputa.pdf

Barral, Catherine; Paterson, Florence; Henri-Jacques Stiker y Michel Chauvière (Eds.) (2000). L'institution du bandicap. Le rôle des associations XIXe-XXe siècle. Rennes, France, PUR.

Bianchi, Eugenia. (2011). Problematizando la noción de trastorno en el TDAH e influencia del manual DSM. Revista Latinoamericana de Ciencias Sociales, Niñezy Juventud, 10 (2), 1021-1038. 
Borzi, Sonia; Sánchez Vázquez, María José y Talou, Carmen (2019). Infancia, discapacidad y educación inclusiva: investigaciones sobre perspectivas y experiencias. La Plata, Argentina. Universidad Nacional de La Plata. Facultad de Psicología. Recuperado de: http://www.psico.unlp.edu.ar/ uploads/docs/infancia_discapacidad_y_educacion_inclusiva.pdf

Borzi, Sonia; Talou, Carmen; Sánchez Vázquez, María José; Hernández Salazar, Vanesa; Gómez, María y Escobar, Silvana (2013). Relevamiento de programas y actividades de atención a la temprana infancia en la ciudad de La Plata. Segunda época, Revista de Psicología (13), 87-99. http://sedici.unlp.edu.ar/handle/10915/43387

Bustelo Graffigna, Eduardo (2012). Notas sobre infancia y teoría: un enfoque latinoamericano. Salud Colectiva, 8(3), 287-298. https://www.redalyc.org/pdf/731/73125097004.pdf

Calfunao, Clarisa; Chavez Asencio, Bárbara; Danel, Paula; Martins, María y Oldani, Karina (2019). "Luchas resemantizadas en contextos neoliberales: discapacidad mercantilizada". En A. Carballeda (Ed.), Dossier Neoliberalismos, democracias y vida pública. Entredichos. Intervenciones y Debates en Trabajo Social. La Plata, Argentina, Facultad de Trabajo Social. Recuperado de: http://entredichos.trabajosocial.unlp.edu.ar/2019/12/13/no7-neoliberalismos-democracias-y-vida-publica/

Castilla García, Claudia (2019). Generaciones e imaginarios sociales en torno a las desigualdades: tensiones y distensiones entre lo instituido y lo instituyente. Material didáctico curso virtual: desigualdades y generaciones en América latina y el Caribe: perspectivas teóricas y experiencias. CLACSO

CEPAL (2010). La pobreza infantil: un desafío prioritario. Desafíos Boletín de la infancia y adolescencia sobre el avance de los objetivos de desarrollo del Milenio. Recuperado de: https:// www.cepal.org/es/publicaciones/35996-la-pobreza-infantil-un-desafio-prioritario

Cobeñas, Pilar (2020). Exclusión educativa de personas con discapacidad: Un problema pedagógico. REICE. Revista Iberoamericana sobre Calidad, Eficacia y Cambio en Educación, 18 (1), 65-81. https://doi.org/10.15366/reice2020.18.1.004

Danel, Paula y Sala, Daniela (2019). Tramas teóricos-metodológicos del trabajo social en el campo gerontológico. Revista Pensamiento y Acción Interdisciplinaria, 5(1), 77-94.

Danel, Paula; Velurtas, Marcela y Favero-Avico, Agustina (2020). Gubernamentalidad neoliberal: miradas desde las intervenciones del Trabajo Social en el Gran La Plata, Argentina. Prospectiva. Revista de Trabajo Social e intervención social, (30), 221-238.

Danel, Paula y Favero Avico, Agustina (2014). Discusiones desde las prácticas sociales sobre la discapacidad, entendida como concepto y campo. Actas IX Jornadas de investigación, docencia, extensión y ejercicio profesional. Facultad de Trabajo Social, UNLP, La Plata, Argentina. Recuperado de: http://sedici.unlp.edu.ar/handle/10915/43728

Danel, Paula (2018). Trabajo social y discapacidad. Intervenciones, trayectorias y temporalidades. Paraná, Argentina, Editorial la hendija.

Dirección General de Cultura y Educación (DGCyE) (2019). Caracterización de la Educación Especial. Recuperado de: http://abc.gob.ar/secretarias/sites/default/files/documents/cracterizacion de la educacion especial.pdf

Dirección General de Cultura y Educación (DGCyE) (2018). Censo Provincial de Matrícula Educativa 2017. Recuperado de: http://www.estadistica.ec.gba.gov.ar/dpe/images/Informe_CPME-2017.pdf 
Dubet, Francois (2015). ¿Por qué preferimos la desigualdad? (aunque digamos lo contrario). Buenos Aires, Argentina, Siglo XXI Editores.

Favero Avico, Agustina (2019). Los Procesos de egreso de jóvenes en situación de institucionalización en la Ciudad de La Plata. Una mirada desde el Trabajo Social. (Tesis de Maestría) Universidad Nacional de La Plata, La Plata, Argentina. Recuperado de: http://sedici.unlp.edu.ar/bitstream/ handle $/ 10915 / 81552 /$ Documento completo.pdf-PDFA.pdf? sequence $=1$ \&isAllowed $=\mathrm{y}$

Fonseca, Claudia y Cardarello, Andrea (1999). Derechos de los más y menos humanos. Horizontes Antropológicos, (10), Año 5.

Foucault, Michel (2011). “Clase del 22 de enero de 1975”. En M. Foucault (Ed.), Los anormales. Buenos Aires, Argentina, Fondo de Cultura Económica.

Grech, Shaun (2016). Discapacidad y Pobreza en la Guatemala Rural: Intersecciones Conceptuales, Culturales y Sociales. Londol School of Higiene and Tropical Medicine. Recuperado de: https://www.lshtm.ac.uk/sites/default/files/2019-06/Guatemala-national-disability-study-Qualitative-Report-spanish.pdf

Grinberg, Julieta (2013). La recepción de "los derechos del niño" en Argentina: trayectorias de activistas y conformación de una nueva causa en torno a la infancia. Virajes, 15 (1), $299 \square 325$.

Gutierrez, Alicia (2004). La construcción social de la pobreza. Un análisis desde las categorías de Pierre Bourdieu. Anduli/Revista Andaluza de Ciencias Sociales, (2), 29-44.

INDEC (2014). Población con dificultad o limitación permanente. Censo Nacional de Población, Hogares y Viviendas 2010. Instituto Nacional de Estadística y Censos, Buenos Aires, Argentina. Recuperado de: https://www.indec.gob.ar/ftp/cuadros/sociedad/PDLP 10 14.pdf

Kaplan, Carina (2006). La inclusión como posibilidad. Ministerio de Educación, Ciencia y Tecnología de la Nación, Buenos Aires, Argentina. Recuperado de: http://www.bnm.me.gov.ar/ giga1/documentos/EL005386.pdf

Ley 24901 Sistema único de prestaciones básicas en habilitación y rehabilitación integral a favor de las personas con discapacidad (1997). Recuperado de: http://servicios.infoleg.gob. ar/infolegInternet/anexos/45000-49999/47677/norma.htm

Ley provincial No 13298 de la Promoción y Protección Integral de los Derechos de los Niños (2007). Recuperado de: http://www.gob.gba.gov.ar/legislacion/legislacion/l-13298.html

Llobet, Valeria (2019). "Las investigaciones en infancia y algunos desafíos para la política y la intervención”. En: A. Barcala y L. Poverene (Eds.) Salud mentaly derechos bumanos en la infancia y adolescencias (pp. 27-32). Remedios de Escalada, Universidad Nacional de Lanús.

Miguez, María Noel (2009). La sujeción de los cuerpos dóciles. Medicación abusiva en la niñez de contexto crítico. XXVII Congreso de la Asociación Latinoamericana de Sociología. VIII Jornadas de Sociología de la Universidad de Buenos Aires. Asociación Latinoamericana de Sociología, Buenos Aires, Argentina. Recuperado de: http://cdsa.aacademica.org/000-062/2180.pdf

Naciones Unidas (2006). Convención sobre los derechos de las personas con discapacidad. Recuperado de: http://www.un.org/esa/socdev/enable/documents/tccconvs.pdf

Oliver, Mike (2002). Capitalismo, discapacidad e Ideología: Una crítica materialista al principio de normalización. Universidad de Greenwich. Recuperado de: http://leeds.ac.uk/disability-studies/ archiveuk/index 
Pinel, Philippe e Itard, Jean (1978). El salvaje del Aveyron: psiquiatría y pedagogia en el iluminismo tardío. Buenos Aires, Argentina, Centro Editor de América Latina.

Provincia de Buenos Aires (2013). Resolución 782/13 Acompañante o Asistente Externo dentro de las instituciones educativas. Recuperado de: http://abc.gob.ar/primaria/sites/ default/files/resolucion_ndeg_782_13_establece_el_accionar_del_acompanante_asistente externo dentro de las instituciones educativas.pdf

Rivas, Silvina; Fischquin, Roxana; Fuentes, Silvana; González, Andrea; Ramacciotti, Cinthya; Roitstein, Gabriela y Vega, Marina (2019). "Subjetividad y Estado: efectos de los procesos de reducción de derecho en el lazo social contemporáneo". En A. Carballeda (Ed.), Dossier Neoliberalismos, democracias y vida pública. Entredichos. Intervenciones y Debates en Trabajo Social. Recuperado de: http://entredichos.trabajosocial.unlp.edu.ar/wp-content/uploads/sites/6/2019/12/Editado-Fischquin-Fuentes-Gonzalez-Ramacciotti-Rivas-Roitstein-y-Vega. pdf

Rodríguez Enriquez, Corina y Marzonetto, Gabriela (2015). Organización social del cuidado y desigualdad: el déficit de políticas públicas de cuidado en Argentina. Revista Perspectivas de Politicas Públicas, (8), 103-134. https://doi.org/10.18294/rppp.2015.949

Sala, Daniela; Pucci, Florencia y Chavez Asencio, Bárbara (2020). "Configuración de identidades colectivas a través de las prácticas políticas en el campo de la discapacidad". En C. Tello y P. Danel (Eds.), Decolonialidad, identidades divergentes e intervenciones (pp. 52-59). La Plata, Argentina, EDULP.

Santos, Milton (2000). Por urna outra globalizacao. Do pensamento único á consciéncia universal. Rio de Janeiro, Brasil, Record.

Segato, Rita (2016). La crítica de la colonialidad en ocho ensayos. Buenos Aires, Argentina, Prometeo Libros.

Segura, Ramiero y Chaves, Marina (2019). "Modos de habitar: localización, tipo residencial y movilidad cotidiana en el Gran La Plata". En M. Di Virgilio y M. Perelman (Eds.), Disputas por el espacio urbano. Desigualdades y territorialidades emergentes (pp. 193 -222). Buenos Aires, Argentina, Editorial Biblos.

Segura, Ramiro (2009). "Si vas a venir a una villa, loco, entrá de otra forma. Distancias sociales, límites espaciales y efectos de lugar en un barrio segregado del gran Buenos Aires". En A. Grimson, C. Ferraudi Curto y R. Segura (Eds.), La vida politica en los barrios populares de Buenos Aires (pp. 41-62). Buenos Aires, Argentina, Prometeo.

Segura, Ramiro (2013). "Los pliegues en la experiencia urbana de la segregación socio-espacial. Análisis comparativo de dos etnografías urbanas". En M. Carman, V. da Cunha y R. Segura (Eds.), Segregación y diferencia en la ciudad (pp. 143-170). Ministerio de Desarrollo Urbano y Vivienda, Ecuador, CLACSO-FLACSO.

Schwamberger, Cintia y Grinberg, Silvia (2020). Devenir escuela colador: dinámicas de escolarización de estudiantes con discapacidad en contexto de pobreza urbana de la Región Metropolitana de Buenos Aires. Praxis Educativa, 24(2), 1-15. https://dx.doi.org/10.19137/ praxiseducativa-2020-240204

Skliar, Carlos (2009). Del miedo al contagio generacional. Revista Todavia, (21) 2-20. http:// www.revistatodavia.com.ar/ 
Vallejos, Indiana (2005). "Parecerse a Nosotros. Debates acerca de las representaciones sociales de los docentes de educación básica acerca de la discapacidad”. En P. Vain y A. Rosato (Eds.), La construcción social de la normalidad Alteridades, diferencias y diversidad. Ensayos y Experiencias. Noveduc, Ciudad de Buenos Aires, Argentina.

Vianna, Adriana (2010). "Derechos, moralidades y desigualdades: consideraciones a partir de procesos de guarda de niños". En C. Villata (Ed.), Infancia, justicia y derechos humanos (pp. 2172). Buenos Aires, Argentina, Editorial de la Universidad Nacional de Quilmes.

Villalta, Carla (2019). "La administración de la infancia y sus familias: un analizador de lo político y lo estatal". En A. Barcala y L. Poverene (Eds.), Salud mental y derechos humanos en la infancia y adolescencias. Buenos Aires, Argentina, Remedios de Escalada de la Universidad Nacional de Lanús.

Walsh, Catherine (2013). Pedagogías decoloniales: Prácticas insurgentes de resistir, (re)existiry (re)vivir. Serie Pensamiento Decolonial. Quito, Ecuador, Abya Yala.

Yuni, José y Meléndez, Cecilia (2017). La puesta en acto de las políticas socioeducativas de inclusión en escuelas secundarias de la Provincia de Catamarca, Argentina. Revista Praxis educativa, 21(1), 55-63. http://dx.doi.org/10.19137/praxiseducativa-2017-210106

A la Mgter. Agustina Favero Avico por la lectura atenta y generosa del artículo. A CONICET por el financiamiento de la investigación y a la Especialización en Infancias y Juventudes (CLACSO) por promover nuevas preguntas y reflexiones. Y, fundamentalmente, a las mamás y a los/as niños y niñas con quienes estuvimos en diálogo, y a los equipos profesionales que aportan a la producción de estata-

Recibido: 01/02/2021

Aceptado: 10/05/2021 